\title{
Lazer e qualidade de vida: considerações frente ao processo de envelhecimento
}

\author{
Leisure and quality of life: considerations about the aging process
}

\author{
Marcelo Martinelli ${ }^{1,4}$, Adriana Munhoz Carneiro ${ }^{2,4}$, Fabián Javier Marín Rueda ${ }^{3,4}$ \\ ${ }^{1}$ Educador Físico, Psicólogo, Mestre em Psicologia com ênfase em Avaliação Psicológica pelo Programa de Pós-Graduação Stricto Sensu em Psicologia; ${ }^{2}$ Psicóloga, Mestre em \\ Psicologia com ênfase em Avaliação Psicológica pelo Programa de Pós-Graduação Stricto Sensu em Psicologia; ${ }^{3}$ Psicólogo, Doutor em Psicologia com ênfase em Avaliação \\ Psicológica, Bolsista de Produtividade do CNPq, Docente do Programa de Pós-Graduação Stricto Sensu em Psicologia; ${ }^{4}$ Universidade São Francisco (USF), Itatiba, SP.
}

\section{RESUMO}

Objetivos: Avaliar a correlação entre lazer e qualidade de vida e investigar diferenças entre idosos e adultos com idade entre 50 e 59 anos. Métodos: Foram incluídos no estudo sujeitos de ambos os gêneros, frequentadores de um centro de convivência para idosos de um município do interior do estado de São Paulo. Foram utilizados os instrumentos Word Health Organization Quality of Life Instrument Bref (WHOQOLBref), versão em português, e a Escala de Avaliação do Lazer (EAVLA). Para verificar a correlação entre os fatores do lazer e domínios da qualidade de vida e diferenças de média entre grupos de diferentes idades, foram utilizadas correlação de Pearson e teste t de Student. Para analisar a influência do lazer na qualidade de vida foi aplicado um modelo de equações estruturais.

Resultados: Participaram 106 sujeitos, divididos em Grupo 1 (50-59 anos) e Grupo 2 (60-82 anos). Na correlação entre lazer em geral e qualidade de vida, o coeficiente de Pearson correspondeu a 0,32 no domínio relações sociais (correlação positiva moderada), 0,28 no do meio ambiente e 0,20 no global (correlações positivas fracas). Por meio do modelo de equações estruturais, o lazer explicou $10 \%$ da qualidade de vida. Não houve diferença na qualidade de vida entre os dois grupos. Nas atividades de lazer socioculturais, o Grupo 1 apresentou média de $88,52 \pm 15,58$, enquanto o Grupo 2 obteve $96,41 \pm 13,37(\mathrm{p}=0,009)$.

Conclusões: As atividades socioculturais obtiveram maior pontuação no grupo com até 59 anos. Em que pese a baixa ou moderada correlação entre os construtos, este estudo confirmou que o lazer foi um componente relevante na qualidade de vida de pessoas com idade entre 50 e 82 anos e sugere aprofundar os estudos com essa população, visando verificar com maior precisão a contribuição mútua entre estes construtos e o manejo de variáveis em processos de intervenção.

DESCRITORES: ENVELHECIMENTO; PROMOÇÃO DA SAÚDE; LAZER; QUALIDADE DE VIDA.

\section{ABSTRACT}

Aims: To evaluate the correlation between leisure and quality of life and to investigate differences between elderly and adults aged between 50 and 59 years.

Methods: The study included subjects of both genders, attendees of a community center for the elderly in a municipality in the state of São Paulo. The instruments Word Health Organization Quality of Life Instrument Bref (WHOQOL-Bref) in its Portuguese version and Leisure Assessment Scale (EAVLA) were used. To verify the correlation between leisure factors and domains of quality of life, as well as differences between the groups with more and less than 60 years old, Pearson correlation and Student $t$ test were used. To analyze the influence of leisure on the quality of life, a model of structural equations was applied.

Results: The study included 106 subjects, divided into Group 1 (50-59 years) and Group 2 (60-82 years). Considering correlation between general leisure and quality of life, the Pearson coefficient corresponded to 0.32 in the social relationships domain (moderate positive correlation), to 0.28 in the environmental domain and to 0.20 in the global quality of life (weak positive correlations). By means of the structural equation model, leisure corresponded to $10 \%$ of quality of life. There was no difference in quality of life between the two groups. In the socio-cultural leisure activities, the Group 1 had an average of 88.52 \pm 15.58 , while Group 2 had $96.41 \pm 13.37$ ( $\mathrm{p}=0.009$ ).

Conclusions: Sociocultural activities scored higher in the group aged up to 59 years. Despite the low or moderate correlation between constructs, this study confirmed that leisure was an important component in the quality of life of people aged between 50 and 82 years and suggests further studies with this population, in order to ascertain more precisely the mutual assistance between these constructs and the handling of variables in intervention processes.

KEY WORDS: AGING; WELLNESS PROGRAMS; LEISURE; QUALITY OF LIFE. 


\section{INTRODUÇÃO}

O século XX trouxe mudanças no âmbito da saúde pública, e o aumento de 30 anos na expectativa de vida da população foi um dos fatores mais significativos para essas transformações. Tal fato produziu uma inversão na pirâmide etária da população, uma vez que as pessoas consideradas idosas e que estavam no topo da pirâmide no início do século passado, agora se inserem no meio dela. ${ }^{1}$ De acordo com o Instituto Brasileiro de Geografia e Estatística, a projeção é que em 2050 haverá 172,7 idosos para cada 100 crianças de 0 a 14 anos. $^{2} \mathrm{O}$ aumento dessa população deve-se a um conjunto de fatores, dentre os quais podem ser citados os avanços científicos, as melhores condições de vida, a diminuição da natalidade, o maior acesso aos serviços de saúde, as campanhas de vacinação, o aumento dos anos de escolaridade e as melhores condições sanitárias e econômicas. . $^{1,34}$

Camarano ${ }^{5,6}$ assinala que, quanto maior a longevidade dos adultos em detrimento dos adolescentes, mais rápido será o envelhecimento populacional. Esta afirmação suscita a necessidade de se olhar para políticas públicas e projetos voltados para o envelhecimento da população e que venham a promover a saúde, principalmente dos idosos. Diante disso, há a necessidade de se buscar fatores que contribuam para uma maior qualidade no envelhecer. Apesar dessa questão se mostrar subjetiva, há uma convergência entre os estudiosos acerca de alguns elementos, que estão relacionados à forma como o sujeito lida com perdas, às boas condições de vida, à criação ou renovação de projetos de vida e às oportunidades de vivências socioculturais. ${ }^{7-9}$ Além destas categorias, existe também a qualidade de vida, que é considerada um conceito básico e central no campo de atenção ao idoso. ${ }^{10}$

Conforme definição do grupo de estudos em avaliação de qualidade de vida da Organização Mundial da Saúde (WHOQOL Group), ${ }_{11}^{11}$ qualidade de vida pode ser entendida como a forma em que o sujeito avalia sua vida segundo sua cultura, sistema de valores, objetivos de vida, expectativas, padrões e preocupações. O conceito qualidade de vida, concebido desta maneira, preconiza a importância de que as pessoas sintam-se bem psicologicamente, estejam socialmente integradas, funcionalmente autônomas e em plenas condições físicas. Ainda, observa-se que, independente da cultura, são obrigatoriamente constituintes desse conceito a subjetividade, a multidimensionalidade e a presença de dimensões positivas (mobilidade) e/ou negativas (dor). ${ }^{12}$ Assim, tem-se um conceito abrangente, que contempla de forma complexa a saúde física e psicológica, níveis de independência e autonomia, relações sociais e componentes pertencentes ao indivíduo e seu ambiente.

Em relação à qualidade de vida, Santos et al. ${ }^{13}$ defendem que o lazer é um construto que merece atenção ao falar do idoso, pois é de suma importância no resgate da saúde e qualidade de vida dos mesmos. De acordo com esses autores, incentivar e proporcionar atividades de recreação mostra-se uma estratégia eficaz para a redução do isolamento, a maximização da inserção do idoso no meio social e, também, para o desenvolvimento de novas habilidades.

O lazer é definido como a dedicação espontânea de tempo para a realização de diferentes atividades, que vão desde repousar e entreter-se, até a participação social voluntária em algum projeto. ${ }^{14} \mathrm{~A}$ importância do lazer na melhoria da qualidade de vida dos idosos tem sido relatada na literatura nos últimos anos. ${ }^{13,15,16}$

Assim, há evidências de que o envelhecimento, a qualidade de vida e o lazer são fenômenos relacionados, uma vez que para obter um envelhecimento bem sucedido, a saúde, a qualidade de vida e o lazer devem estar presentes. Dentro deste contexto, este estudo teve como objetivo investigar diferenças entre idosos e adultos com 50 anos de idade ou mais, utilizando dois instrumentos que oferecem medidas confiáveis de qualidade de vida e lazer. Adicionalmente buscouse investigar a correlação entre esses construtos, quantificando a influência do lazer na qualidade de vida da amostra em estudo.

\section{MÉTODOS}

O delineamento da pesquisa foi transversal e quantitativo. Inicialmente houve a aprovação do Comitê de Ética da Universidade São Francisco sob o Parecer Consubstanciado $\mathrm{n}^{\circ} 76296$ e, posteriormente, todos os participantes assinaram o Termo de Consentimento Livre e Esclarecido.

Os participantes da pesquisa são frequentadores do Centro de Convivência do Idoso do município de Itatiba, no estado de São Paulo, gerido pelo Fundo Social de Solidariedade, o qual possui parceria com a Universidade São Francisco por meio do primeiro termo aditivo ao convênio técnico-didático-científico, firmado em 01/09/2009, que visa promover a relação entre a universidade e a comunidade da terceira idade por meio do desenvolvimento de atividades de Extensão Universitária com área temática em saúde. A coleta de dados foi realizada em um salão comunitário, local onde são desenvolvidas diversas atividades, dentre elas, aulas de ginástica, Tai-Chi Chuan, bingo, 
bailes e ensaios do coral. Foram alocadas mesas e cadeiras para a viabilização do preenchimento dos instrumentos. Após esclarecimentos gerais sobre a pesquisa e a forma de responder os instrumentos, os dados foram coletados em duas sessões, cada uma com duração de aproximadamente 30 minutos.

Considerando o parâmetro da Organização Mundial de Saúde ${ }^{17}$ e o Estatuto do Idoso, ${ }^{18}$ deve ser considerado como idoso a pessoa acima de 60 anos nos países em desenvolvimento. Assim, nas análises estatísticas realizadas, os participantes foram divididos em dois grupos: Grupo 1 (sujeitos de 50 até 59 anos) e Grupo 2 (de idosos, entre 60 e 82 anos). Embora o Grupo 2 esteja fora da classificação da OMS para idosos, essas pessoas são aceitas nas atividades oferecidas pelo Centro de Convivência do Idoso. Todos os participantes eram alunos praticantes do curso de Tai-Chi Chuan, realizando essa atividade duas vezes por semana, com duração de sessenta minutos por sessão, há pelo menos um ano.

Para a coleta de dados foram utilizados o Word Health Organization Quality of Life Instrument Bref (WHOQOL-Bref) em sua versão em português, e a Escala de Avaliação do Lazer (EAVLA). A escolha desses instrumentos deu-se com base nas qualidades métricas, que atestam sua precisão e validade.

O WHOQOL-Bref ${ }^{19}$ é uma versão reduzida do WHOQOL-100, utilizado para avaliar a qualidade de vida relacionada à saúde do adulto em grupos de pessoas sadias ou enfermas, incluindo idosos. A escala é auto aplicável e possui 26 questões, sendo duas gerais sobre qualidade de vida e 24 relacionadas aos quatro domínios avaliados: físico, psicológico, relações sociais e meio ambiente.

A EAVLA ${ }^{20}$ tem como base a concepção de lazer apresentada por Dumazedier, ${ }^{21}$ Gaelzer $^{22}$ e Loureiro, ${ }^{23}$ e se destina a avaliar o lazer de pessoas adultas. O instrumento também é auto aplicável, e possui 53 itens referentes a atividades de lazer, que devem ser respondidos de acordo com uma escala que vai de "não gosto" a "gosto". A escala inclui três fatores: atividades socioculturais (25 itens), atividades artístico-manuais e criativas (15 itens), e atividades físico-esportivas (13 itens). A soma dos três fatores oferece a medida de Lazer Geral.

Os dados foram analisados pelo programa Statistical Package Social Science-SPSS-Versão 15, e pelo AMOS 7, contido no SPSS. As análises inferenciais tiveram como objetivo verificar a correlação entre os fatores do lazer e domínios da qualidade de vida e diferenças de média entre os grupos com idade inferior e acima de 60 anos. Para tal, foram utilizadas estatísticas paramétricas bivariadas (correlação de
Pearson e teste $t$ de Student). Para classificação da magnitude encontrada nas correlações foi utilizado o critério de Dancey e Reidy. ${ }^{24}$ Para analisar a influência do lazer na qualidade de vida foi utilizado um modelo de equações estruturais considerando ambas as escalas e os respectivos fatores latentes de cada construto. $\mathrm{O}$ ajuste do modelo foi estudado por meio dos índices Qui $^{2} /$ Graus de Liberdade $\left(\chi^{2} / \mathrm{gl}\right)$, Goodness-of-fit Statistic (GFI), Comparative Fit Index (CFI), Root Mean Square Error of Approximation (RMSEA) e Standardized Root Mean Square Residual (SRMR), apontados pela literatura como os mais importantes. ${ }^{25-27}$

\section{RESULTADOS}

Fizeram parte do estudo 106 sujeitos com idade mínima de 50 e máxima de 82 anos (mediana 62; média $62,79 \pm 7,57)$, sendo $93(87,7 \%)$ participantes do gênero feminino. O Grupo 1 foi composto por 67 sujeitos com idade mínima de 50 e máxima de 59 anos (mediana 56; média 55,31 $\pm 3,01)$, sendo $60(89,6 \%)$ mulheres. O Grupo 2 foi composto por 39 sujeitos, com idade mínima de 60 e máxima de 82 anos (mediana 66; média $67,15 \pm 5,80)$, sendo $33(84,6 \%)$ mulheres. Embora não tenha sido investigada a condição de saúde da população estudada, destaca-se que todos os membros do Centro de Convivência do Idoso mostravam-se pessoas ativas, não relatando nenhuma doença que lhes impossibilitasse realizar qualquer atividade das propostas pelo projeto.

Primeiramente foi realizado o teste $t$ de Student para observar se havia diferenças entre as médias do Grupo 1 e Grupo 2 no WHOQOL-Bref e na EAVLA. Das nove variáveis estudadas em ambos os instrumentos, encontrou-se valor estatisticamente significativo apenas para o fator de atividades de lazer socioculturais, indicando que o grupo com menos de 60 anos apresentou média superior (Tabela 1).

$\mathrm{Na}$ sequência, os instrumentos foram correlacionados considerando os domínios e fatores de cada um deles e a pontuação geral. Pelo resultado da análise correlacional observa-se que o domínio relações sociais do WHOQOL-Bref apresentou correlações positivas e estatisticamente significativas com todas as medidas da EAVLA. Em que pese a isso, as magnitudes são consideradas fracas, revelando que a variância comum das dimensões avaliadas por esses instrumentos é pequena. Importante destacar que a magnitude fraca foi observada em todas as correlações que foram estatisticamente significativas. Ainda, destaca-se que o domínio psicológico do WHOQOL-Bref não teve resultados estatisticamente significativos com nenhuma medida da EAVLA (Tabela 2). 
Tabela 1. Diferença de média na qualidade de vida e no lazer para os grupos de pessoas adultas entre 50 e 59 anos e de idosos entre 60 e

82 anos, participantes de um centro de convivência para idosos em Itatiba, SP.

\begin{tabular}{|c|c|c|c|c|c|c|}
\hline Domínios WHOQOL-Bref & Grupo & N & Média & DP & $t$ & $p$ \\
\hline \multirow[t]{2}{*}{ Físico } & até 59 anos & 39 & 15,81 & 2,67 & \multirow{2}{*}{0,77} & \multirow{2}{*}{0,444} \\
\hline & 60 anos ou mais & 67 & 15,39 & 2,78 & & \\
\hline \multirow[t]{2}{*}{ Psicológico } & até 59 anos & 39 & 15,06 & 2,09 & \multirow{2}{*}{$-1,33$} & \multirow{2}{*}{0,185} \\
\hline & 60 anos ou mais & 67 & 15,66 & 2,32 & & \\
\hline \multirow[t]{2}{*}{ Relações Sociais } & até 59 anos & 39 & 15,04 & 2,96 & \multirow{2}{*}{0,70} & \multirow{2}{*}{0,483} \\
\hline & 60 anos ou mais & 67 & 14,57 & 3,56 & & \\
\hline \multirow[t]{2}{*}{ Meio Ambiente } & até 59 anos & 39 & 15,09 & 2,25 & \multirow{2}{*}{$-0,49$} & \multirow{2}{*}{0,625} \\
\hline & 60 anos ou mais & 67 & 15,31 & 2,27 & & \\
\hline \multirow[t]{2}{*}{ Global } & até 59 anos & 39 & 16,10 & 2,47 & \multirow{2}{*}{0,51} & \multirow{2}{*}{0,608} \\
\hline & 60 anos ou mais & 67 & 15,76 & 3,69 & & \\
\hline \multicolumn{7}{|l|}{ Atividades de lazer } \\
\hline \multirow[t]{2}{*}{ Socioculturais } & até 59 anos & 39 & 96,41 & 13,37 & \multirow{2}{*}{2,65} & \multirow{2}{*}{0,009} \\
\hline & 60 anos ou mais & 67 & 88,52 & 15,58 & & \\
\hline \multirow[t]{2}{*}{ Artístico-manuais e criativas } & até 59 anos & 39 & 36,51 & 11,88 & \multirow{2}{*}{0,03} & \multirow{2}{*}{0,976} \\
\hline & 60 anos ou mais & 67 & 36,43 & 13,69 & & \\
\hline \multirow[t]{2}{*}{ Físico-esportivas } & até 59 anos & 39 & 27,46 & 10,59 & \multirow{2}{*}{1,24} & \multirow{2}{*}{0,216} \\
\hline & 60 anos ou mais & 67 & 25,04 & 9,06 & & \\
\hline \multirow[t]{2}{*}{ Geral } & até 59 anos & 39 & 160,38 & 28,12 & \multirow{2}{*}{1,69} & \multirow{2}{*}{0,093} \\
\hline & 60 anos ou mais & 67 & 150,00 & 31,72 & & \\
\hline
\end{tabular}

WHOQOL-Bref: Word Health Organization Quality of Life Instrument Bref. DP: desvio padrão; $t$ : Teste T.
Tabela 2. Correlação de Pearson entre os domínios do Word Health Organization Quality of Life Instrument Bref (WHOQOL-Bref), versão em português, e a Escala de Avaliação do

Lazer (EAVLA), utilizando a amostra total de 106 pessoas entre 50 e 82 anos, participantes de um centro de convivência para idosos em Itatiba, SP.

\begin{tabular}{lccccc} 
& \multicolumn{5}{c}{ Domínios do WHOQOL-Bref } \\
\cline { 2 - 6 } \multicolumn{1}{c}{ Fatores da EAVLA } & Físico & Psicológico & $\begin{array}{c}\text { Relações } \\
\text { Sociais }\end{array}$ & $\begin{array}{c}\text { Meio } \\
\text { Ambiente }\end{array}$ & Global \\
Socioculturais & $0,30^{* *}$ & 0,14 & $0,33^{* *}$ & $0,29^{* *}$ & $0,28^{* *}$ \\
Artístico-manuais e criativas & 0,14 & 0,07 & $0,20^{*}$ & $0,20^{*}$ & 0,12 \\
Físico-esportivas & $-0,03$ & $-0,02$ & $0,21^{*}$ & 0,15 & 0,03 \\
Geral & 0,20 & 0,09 & $0,32^{* *}$ & $0,28^{* *}$ & $0,20^{*}$ \\
\hline
\end{tabular}

* Significativo ao nível de 0,05; ** significativo ao nível de 0,01.
Dando continuidade, estudou-se a relação entre os instrumentos por meio de um modelo de equações estruturais, verificando o quanto da qualidade de vida dessa amostra poderia ser explicada pelo lazer. A Figura 1 apresenta o modelo testado.

Nos índices de ajuste do modelo foi observado um valor de $\chi^{2} / \mathrm{gl}=1,72$, que não foi estatisticamente significativo ( $\mathrm{p}=0,051)$, ou seja, aceitou-se a hipótese nula de que a matriz observada e a matriz do modelo não são significativamente diferentes, ou que são muito próximas. Ainda, os valores de GFI, CFI, RMSEA e SMR foram 0,95, 0,95, 0,08 e 0,07 respectivamente, que são considerados muito adequados, ou seja, a estrutura psicométrica do WHOQOL-Bref e da EAVLA foram confirmados com dados diferentes, indicando a estabilidade de sua estrutura. Observou-se também que o coeficiente de correlação de Pearson entre as variáveis latentes de lazer e qualidade de vida foi 0,32 , e que $10 \%$ da qualidade de vida pôde ser explicada pelo lazer.

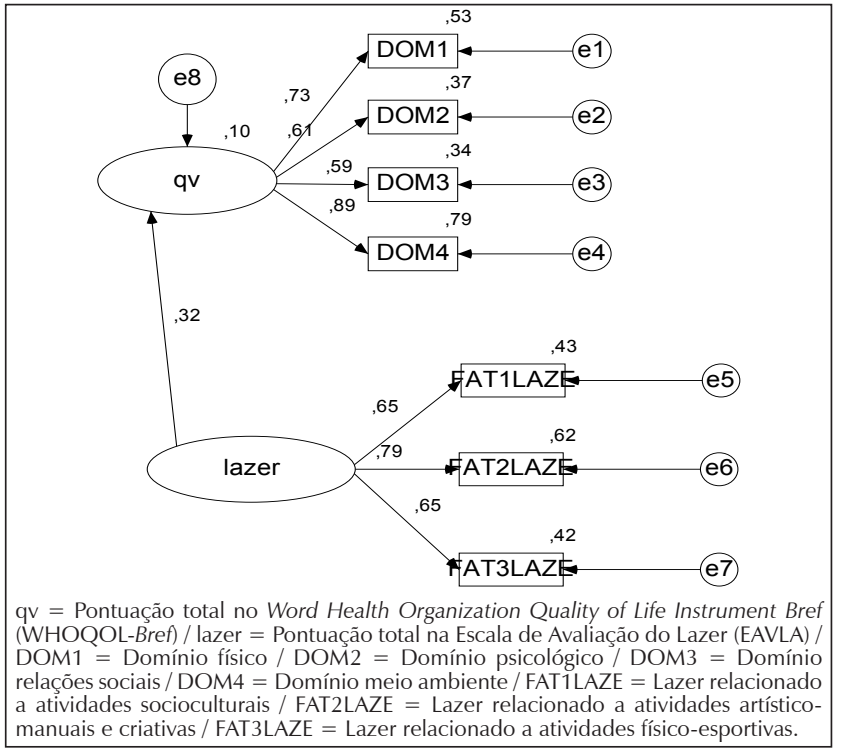

Figura 1. Modelo de equações estruturais para a WHOQOLBref e a EAVLA. 


\section{DISCUSSÃO}

A proposta desta pesquisa foi estudar a relação entre lazer e qualidade de vida em um grupo de pessoas que frequentavam um centro de convivência do idoso. Primeiramente, e considerando o fator diferença de idade entre os grupos, houve diferença estatisticamente significativa apenas para as atividades de lazer socioculturais, sendo que o grupo mais jovem obteve maior pontuação. Este dado pode indicar que os participantes do Grupo 1 ( 50 a 59 anos), por serem mais jovens, gozam de maior autonomia e capacidade funcional em relação aos participantes do Grupo 2 (60 a 82 anos) e, portanto, relacionam-se de maneira mais eficiente e satisfatória com o meio ambiente em situações que exijam participação ativa, interatividade e funcionalidade. Diante disso, podemos inferir uma tendência dos mais jovens a preferirem atividades desse fator, quais sejam, viajar, ir a restaurantes, ir ao cinema, navegar na internet, participar de festas familiares, ir ao teatro, frequentar bailes, receber os amigos em casa, entre outras. Analisando as demais médias, também se observou que o grupo com menos de 60 anos, de modo geral, obteve as maiores pontuações, embora essas diferenças não tenham sido estatisticamente significativas. Este dado é importante, haja vista que o processo de senescência, mesmo sem o acometimento por doenças, gera perdas funcionais, como diminuição da acuidade visual e auditiva, dificuldade de locomoção, retraimento psicológico, maior vulnerabilidade a depressão entre outros fatores, que impõem barreiras concretas ao idoso em suas relações pessoais e socioculturais..$^{9,13,28}$

No que se refere às relações entre os construtos, houve correlação positiva e estatisticamente significativa entre o Domínio Físico do WHOQOL-Bref e as atividades de lazer socioculturais da EAVLA. O Domínio Físico avalia a capacidade funcional dos indivíduos idosos, como locomoção, energia para enfrentar o dia-a-dia, nível de dor (física), uso de medicamentos, sono e repouso, capacidade para o trabalho e tratamento médico. Diante disso, pode-se considerar que as atividades praticadas no Centro de Convivência do Idoso podem ter contribuído para que essa população tenha participado e estado afetivamente envolvida em atividades de lazer socioculturais, ou seja, quanto mais qualidade de vida o indivíduo percebe em si próprio no Domínio Físico, mais se aproxima sócio culturalmente por meio de afetos positivos de seus pares, fazendo uso de sua autonomia e funcionalidade. Por outro lado, de acordo com Santos et al., ${ }^{13}$ o lazer, somado ao exercício, às atividades mentais e à manutenção das relações sociais, tem papel protetor sobre as funções cognitivas na velhice, conferindo assim melhora na qualidade de vida. Dessa forma, a correlação não revela qual é a causa e qual é o efeito.

O Domínio Relações Sociais correlacionou-se positiva e significativamente com todos os fatores da EAVLA. Esse domínio mensura o quão satisfeito o indivíduo está com suas relações pessoais, relações sexuais e apoio dos amigos. Este resultado indica a boa relação social da população estudada com as atividades de lazer de um modo geral, e também da importância qualitativa das relações pessoais, sociais e de apoio dos amigos, para que o idoso se aproxime de atividades salutares para o seu benefício. Os idosos, segundo Davim et al., ${ }^{15}$ procuram atividades de lazer para se entreter e evitar momentos de solidão, por meio da participação em grupos, viagens, bailes, oficinas de artesanato, dentre outros, na busca de experiências significativas e afetuosas. Essas experiências podem satisfazer as expectativas e atender às necessidades do idoso, conduzindo-o a uma ressignificação emocional do lazer. ${ }^{16}$

Por sua vez, as atividades de lazer socioculturais, artístico-manuais e criativas, assim como o lazer geral, tiveram correlação positiva e estatisticamente significativa com o Domínio Meio Ambiente. Este domínio avalia o quanto o sujeito se sente satisfeito em relação à segurança física e proteção, ambiente no lar, recursos financeiros, cuidados de saúde e sociais, quanto à disponibilidade e qualidade, oportunidades de adquirir novas informações e habilidades, participação em oportunidades de recreação/lazer, ambiente físico (poluição/ruído/trânsito/clima) e transporte. ${ }^{29}$ Este resultado mostra que as atividades de lazer em questão tendem a ser mais vivenciadas por esta população na medida em que as variáveis do meio ambiente são atendidas e vividas num contexto qualitativo.

No que se refere ao Domínio Psicológico do WHOQOL-Bref, ele não se correlacionou significativamente com nenhuma das atividades de lazer da EAVLA. A esse respeito, pode-se mencionar que, do ponto de vista funcional e orgânico, o idoso tem reduzida mobilidade, equilíbrio, atividade do sistema nervoso e a reserva funcional cardíaca. ${ }^{9,28,30} \mathrm{~A}$ senescência, como também o envelhecimento patológico, reduz capacidades cognitivas como memória, concentração, velocidade do raciocínio, modifica a aparência e o conceito de imagem corporal e pode facilitar o aparecimento de sentimentos negativos e aumentar a vulnerabilidade à depressão. Essas perdas físicas, e especificamente psicológicas, contribuem negativamente para que os idosos vivenciem seus momentos de lazer. Como defendem Teixeira e 
Neri, ${ }^{8}$ dentre os componentes mais importantes para que se obtenha um envelhecimento bem sucedido, o aspecto subjetivo é o mais importante, característica essa do Domínio Psicológico do WHOQOL-Bref. Diante disso, pode-se inferir que a falta de correlação significativa com o domínio psicológico se deu por conta das perdas anteriormente mencionadas e o quão importante se faz, ao se pensar em propor atividades e programas destinados aos idosos, que sejam eficazes na promoção e resgate da autoestima, subjetividade e demais aspectos psicológicos.

Por fim, o domínio WHOQOL-Geral obteve correlação positiva e estatisticamente significativa com as atividades dos fatores Sociocultural e Lazer Geral da EAVLA. Essa correlação mostra proximidade entre os construtos estudados, especialmente nos momentos de socialização e cultura, ou seja, quanto mais afetividade e participação em atividades de lazer, mais contribuições serão dadas à qualidade de vida da população idosa e vice versa. Santos et al. ${ }^{13}$ defendem que atividades de recreação e lazer proporcionam autoestima, melhora na condição de saúde e na qualidade de vida do idoso.

Importante se faz mencionar que o Domínio WHOQOL-Geral e o Fator Lazer Geral, que representam a soma total dos instrumentos utilizados, obtiveram correlação estatisticamente significativa e de baixa magnitude $(r=0,20)$, explicada anteriormente pela multidimensionalidade do constructo qualidade de vida. Também deve ser ressaltado que, ao considerar os instrumentos livres de erro, como feito pela modelagem de equações estruturais, essa correlação aumentou para 0,32 , o que indica que, de fato, há relação entre o lazer e a qualidade de vida. Além disso, o lazer explicou $10 \%$ da qualidade de vida. Com isso, e como menciona o WHOQOL Group,${ }^{11}$ embora a qualidade de vida se apresente como um constructo subjetivo e multidimensional, e o lazer não abarque todas as dimensões desse constructo, ele contribui positivamente e de maneira diferenciada para cada indivíduo no que diz respeito à sua qualidade de vida.
Para os idosos, a qualidade de vida é percebida como a valorização da questão afetiva e familiar, a obtenção de prazer e conforto, assim como uma forma de colocar em prática seu ideário de vida. Diante disso, os exercícios físicos, atividades cognitivas e o cultivo das relações sociais, inseridos num contexto de lazer, tem função protetora na qualidade de vida do idoso. Gradualmente, por meio da informação e a participação em programas de lazer e qualidade de vida destinados aos idosos, essa população vem se conscientizando de seus direitos legais e de participação ativa na sociedade, deixando de lado imagens de apatia, sedentarismo, subserviência e tristeza, observando-se cada vez mais a presença do idoso em cada parque, cinema, teatro, escola, família, partidos políticos, igrejas, dentre tantos outros locais e segmentos sociais. Esse fato chama a atenção da sociedade para a reversão de seu papel de cidadão passivo na busca de um significado mais ativo para sua vida.

Este estudo buscou contribuir com os estudos de lazer e qualidade de vida referentes às pessoas com 50 anos ou mais de idade, confirmando que o lazer foi um componente relevante na qualidade de vida dessas pessoas, e que as atividades socioculturais, que exigem mais interatividade e funcionalidade, obtiveram maior pontuação no grupo com menos de 60 anos. Como limitações, este estudo limitou-se ao delineamento transversal, não buscando uma possível explicação de causa e efeito na correlação entre os construtos. A amostra foi modesta e predominantemente feminina, como também a comparação entre grupos se deu entre pessoas participantes das atividades oferecidas pelo Centro de Convivência do Idoso, e não com um grupo controle de não participantes, o que poderá ser estudado futuramente. Diante da complexidade e abrangência do tema estudado, sugere-se que outras pesquisas sejam feitas, considerando aspectos sociais e demográficos, utilização de mais instrumentos que mesurem lazer e qualidade de vida e delineamentos longitudinais, com o intuito de verificar com maior precisão a contribuição mútua entre estes construtos e o manejo de variáveis em processos de intervenção.

\section{AGRADECIMENTOS}

À prefeitura municipal de Itatiba por meio do Fundo Social de Solidariedade e ao Núcleo de Extensão Universitária da Universidade São Francisco, que, em parceria, são promotores das atividades realizadas para os idosos onde foi permitida a coleta de dados deste estudo. Especialmente, agradecer aos idosos participantes desta pesquisa.

\section{REFERÊNCIAS}

1. Abdo C. Estudo populacional do envelhecimento no Brasil. São Paulo: Segmento Farma; 2009.

2. Instituto Brasileiro de Geografia e Estatística (IBGE). Projeção da população do Brasil por sexo e idade: 1980-2050. Rio de Janeiro: IBGE; 2008. 
3. Veras R. A era dos idosos: novos desafios. In: Anais da Primeira Oficina de trabalho sobre desigualdades sociais e de gênero em saúde de idosos no Brasil. Ouro Preto: Núcleo de Estudos em Saúde Pública e Envelhecimento, Centro de Pesquisas René Rachou da Fundação Oswaldo Cruz, Faculdade de Medicina da Universidade Federal de Minas Gerais; 2002.

4. Veras RP, Caldas CP. Promovendo a saúde e a cidadania do idoso: o movimento das universidades da terceira idade. Ciênc Saúde Colet. 2004;9:423-32.

5. Camarano AA, organizador. Muito além dos 60: os novos idosos brasileiros. Rio de Janeiro: Ipea; 1999.

6. Camarano AA. Envelhecimento da população brasileira: uma contribuição demográfica. Rio de Janeiro: Ipea; 2002.

7. Caldas PC. Quarta idade: a nova fronteira da gerontologia. In: Papaléo Netto M, organizador. Tratado de gerontologia. São Paulo: Atheneu; 2007. p. 339-41.

8. Teixeira I, Neri AL. Envelhecimento bem-sucedido: uma meta no curso da vida. Psicol USP. 2008;19:81-94.

9. Yuaso DR, Netto MP. Como envelhecer bem? São Paulo: Paulus; 2009

10. Castellón ASP, Rubio RH, Aleizandre MR. Nivel de autoestima en los mayores tras un programa de ocio. Rev Esp Geriatr Gerontol. 1999;34(4):225-9.

11. Fleck MPA, Leal OF, Louzada S, Xavier M, Chachamovich E, Vieira G, Santos L, Pinzon V. Desenvolvimento da versão em português do instrumento de avaliação de qualidade de vida da OMS (WHOQOL-100). Rev Bras Psiquiatr. 1999;21:19-28.

12. Moreno AB, Faerstein E, Werneck GL, Lopes CS, Chor D. Propriedades psicrométricas do instrumento abreviado de avaliação de qualidade de vida da Organização Mundial de Saúde no estudo Pró-Saúde. Cad Saúde Públ. 2006;22:2585-97.

13. Santos PL, Foroni PM, Chaves MCF. Atividades físicas e de lazer e seu impacto sobre a cognição no envelhecimento. Med Rib Preto. 2009; $42: 54-60$

14. Dumazidier J. Lazer e cultura popular. São Paulo: Perspectiva; 1973.

15. Davim RMB, Dantas SMM, Lima VM, Lima JFV. O Lazer diário como fator de qualidade de vida: o que pensa um grupo da terceira idade. Ciênc Cuid Saúde Maringá. 2003;2:19-24.

16. Gáspari JC, Schwartz GM. O idoso e a ressignificação emocional do lazer. Psic.: Teor. e Pesq. 2005;21:69-76.

17. Organização Mundial de Saúde. Envelhecimento ativo: uma política de saúde. Brasília: Organização Pan-Americana da Saúde; 2005.

18. Brasil. Estatuto do idoso: lei federal no 10.741, de 01 de outubro de 2003. Brasília: Secretaria Especial dos Direitos Humanos; 2004.

19. Fleck MPA, Louzada S, Xavier M, Chachamovich E, Vieira G, Santos L, et al. Aplicação da versão em português do instrumento abreviado de avaliação da qualidade de vida "WHOQOL-Bref”. Rev Saúde Públ. 2000;34(2):178-83.

20. Martinelli M. Escala de Avaliação do Lazer (EAVLA). Itatiba: Universidade São Francisco; 2011.

21. Dumazidier J. Valores e conteúdos culturais do lazer. São Paulo: Sesc; 1980.

22. Gaelzer L. Lazer: benção ou maldição. Porto Alegre: Sulina; 1979.

23. Loureiro SMM. O lazer na vida de estudantes de pós-graduação de psicologia e educação física na universidade de São Paulo: Um estudo exploratório [Dissertação]. [São Paulo]: Universidade de São Paulo; 1995.

24. Dancey CP, Reidy J. Estatística sem matemática para psicologia usando SPSS para Windows. Porto Alegre: Artmed; 2006.

25. Arbuckle JL. Amos 18 User's Guide. Chicago: SPSS Inc; 2009.

26. Byrne B. Structural equation modeling with AMOS: basic concepts, applications, and programming. New York: Psyclology Press, Taylor \& Francis Group; 2001.

27. Tabachnick BG, Fidell LS. Using Multivariate Statistics. Needham Heights: Allyn \& Bacon; 2001.

28. Nahas MV. Atividade física, saúde e qualidade de vida: conceitos e sugestões para um estilo de vida ativo. Londrina: Mediograf; 2006.

29. Guimarães MLR, Hilgert JB, Hugo FN, Corso AC, Nocchi P, Padilha DMP. Impacto da perda dentária na qualidade de vida de idosos independentes. Sci Med. 2005;15(1):30-3.

30. Timm LA, Argimon IL, Wendt GW. Correlação entre domínios de qualidade de vida e lócus de controle da saúde em idosos residentes na comunidade. Sci Med. 2011;21(1):9-13. 